\title{
Caracterização de solos sob diferentes usos na região sul do Amazonas
}

\author{
Ivanildo Amorim de OLIVEIRA ${ }^{1}$, Milton César Costa CAMPOS $^{2 *}$, Ludmila de FREITAS ${ }^{1}$, \\ Marcelo Dayron Rodrigues SOARES ${ }^{3}$ \\ 1 Universidade Estadual Paulista - UNESP, Faculdade de Ciências Agrárias e Veterinárias, Departamento de Solos e Adubos. Via de Acesso Prof. Paulo Donato Castellane. \\ CEP.: 14870-900, Jaboticabal, SP, Brasil. \\ 2 Universidade Federal do Amazonas. Departamento de Agronomia. Rua 29 de Agosto, 786, Centro, CEP.: 69.800-000, Humaitá-AM. \\ ${ }^{3}$ Universidade Federal do Acre - UFAC, Programa de Pós-graduação em Ciência, Inovação e Tecnologia para a Amazônia - CITA. Rod. BR 364, km 04, B. Distrito Industrial, \\ CEP.: 69915-900, Rio Branco, AC, Brasil. \\ * Autor correspondente: mcesarsolos@gmail.com
}

\begin{abstract}
RESUMO
Áreas com vegetação nativa são cada vez mais fragmentadas devido ao desmatamento associado à atividade agropecuária, o que provoca alteraçóes nos atributos químicos, físicos e biológicos do solo. Assim, o objetivo do estudo foi avaliar os atributos físicos e químicos do solo por meio de técnicas estatísticas multivariadas. $\mathrm{O}$ estudo foi realizado em áreas de terra preta arqueológica (TPA), floresta, pastagem, agrofloresta, cana-de-açúcar e mandioca. Nestas áreas foram coletadas 64 amostras de solos deformadas e indeformadas com espaçamento regular de $10 \mathrm{~m}$ na profundidade de $0,0-0,10 \mathrm{~m}$ para determinação dos atributos químicos e físicos. O estudo mostra que as áreas com TPA e agrofloresta apresentaram valores de atributos físicos e químicos diferentes da pastagem, floresta, mandioca e cana-de-açúcar. Por serem ambientes cultivados, as áreas de mandioca, cana-de-açúcar e pastagem apresentam características físicas e químicas similares. O uso das técnicas de análises multivariadas foi eficiente para verificar as similaridades ou as diferenças, com base nos atributos químicos e físicos do solo em cada área estudada.
\end{abstract}

PALAVRAS-CHAVE: análise multivariada, componente principal, solos da Amazônia, terra preta arqueológica.

\section{Characterization of soils under different land uses in the southern region of the Amazonas}

\section{ABSTRACT}

Areas with native vegetation are increasingly fragmented due to agricultural occupation, and with the removal of the natural vegetation, the use of soil has shown changes in their chemical, physical and biological attributes. Thus, the objective of this work was to evaluate soil physical and chemical attributes using multivariate technique. The study was carried out in area with Archaeological Black Earth (TPA), forest, pasture, agroforestry, sugar cane and cassava. In these areas 64 soil samples were collected from disturbed and undisturbed soils at a regular spacing of 10 meters and 0.0 to $0.10 \mathrm{~m}$ depth for chemical and physical analyses. Areas of TPA and agroforestry had different values for physical and chemical attributes in comparison with soils from pasture, forest, cassava and sugar cane. The areas cultivated with cassava, sugar cane and pasture had similar physical and chemical characteristics. The use of the multivariate analysis tool proved to be useful to detect similarities or differences in soils attributes based on chemical and physical in the study area.

KEYWORDS: multivariate analysis, principal component, Amazonian of soils, archaeological black earth. 


\section{INTRODUÇÃO}

A regiāo sul do Amazonas é caracterizada por apresentar ambientes naturais e em uso agropecuário. As áreas com floresta ainda naturais ocupam a maior parte e na tipologia vegetal de florestas da regiâo, destacam-se as Florestas Tropicais Abertas e Densas (Brasil 1978; Campos 2009). Já as áreas cultivadas com cana-de-açúcar apresentam importância social e econômica na produção de álcool e açúcar, bem como na geraçẫo de empregos diretos e indiretos (Marçal 2009) em pequenas indústrias de produção de melaço, rapadura, aguardente e forragem para alimentação animal.

Outros usos agropecuários do solo como o cultivo de mandioca (Manihot sculenta) na região, em geral são em pequena escala, porém, segundo Matos e Bezerra (2003) o seu cultivo tem papel importante, tanto como fonte de alimento como geradora de emprego e renda. Já os ambientes agroflorestais são uma forma de uso da terra na regiâo, na qual as espécies lenhosas perenes (arbustos ou árvores) são cultivadas deliberadamente com espécies agrícolas e/ou com animais, visando estabelecer sustentabilidade ambiental, socioeconômica e cultural (Macdicken e Vergara 1990). Por outro lado, se destaca áreas com terras pretas arqueológicas (TPA), que são solos de alta fertilidade natural e possui como característica marcante a coloração escura e presença de fragmentos de cerâmica e/ou líticos incorporados a matriz dos horizontes superficiais (Kämpf e Kern 2005).

Em áreas com cultivos agrícolas, em razão de alguns processos que levam ao empobrecimento das características físicas, químicas e biológicas, provoca degradação do solo (Silva et al. 2005). O conhecimento das alteraçóes nos atributos do solo, causado pelo cultivo contínuo, fornece subsídios para a adoção de práticas de manejo que permitam incrementar o rendimento das culturas, a contínua sustentabilidade e conservaçáo dos ecossistemas.

Neste sentido, o uso de técnica da análise multivariada é possível explicar o máximo de intercorrelação entre as variáveis e descobrir quais delas contribuem mais para a caracterização e, ou, alteração do solo. $\mathrm{Na}$ análise simultânea de muitas informaçôes, esta técnica torna-se a melhor ferramenta, possibilitando obter dados e interpretaçóes que poderiam não ser perceptíveis com o uso da análise estatística univariada (Cruz e Regazzi 2001).

$\mathrm{O}$ uso conjunto de técnicas multivariadas pode auxiliar na tomada de decisáo do uso e manejo adequado do solo, baseando-se no comportamento dos seus atributos, e indicar os atributos que sofrem maior alteração com a ação antrópica. Assim, o objetivo foi avaliar conjuntamente os atributos físicos e químicos do solo por meio técnicas estatísticas multivariadas na caracterização de ambientes com diferentes usos na regiáo Sul do Amazonas.

\section{MATERIAL E MÉTODOS}

O estudo foi realizado em propriedades rurais, localizadas ao sul do Estado do Amazonas, nos municípios de Manicoré e Humaitá, AM. Foi realizado o mapeamento de seis áreas com diferentes usos: solo em floresta situada nas coordenadas $7^{\circ}$ $54^{\prime} 44,5^{\prime}$ 'S e 61०31'44,7" W (Figura 1) com altitude média de $140 \mathrm{~m}$, caracterizada por apresentar fragmento de Floresta Tropical Densa, formada por árvores de 20 a $50 \mathrm{~m}$ de altura.

Solo com presença de horizonte antrópico ou terra preta arqueológica (TPA), situada nas coordenadas geográficas $7^{\circ}$ $55^{\prime} 02,1^{\prime \prime}$ S e 61०31' 45,2" W numa altitude de $102 \mathrm{~m}$ sendo esta, cultivada com milho com aproximadamente 120 dias após o plantio.

Área de pastagem nas coordenadas $07^{\circ} 54^{\prime} 42^{\prime \prime} \mathrm{S}$ e $61^{\circ}$ 31 ' 50 " W, altitude média de $135 \mathrm{~m}$ com uso de braquiária (Brachiaria brizanta) com 10 anos em pastejo extensivo e a capacidade de suporte de animais de uma (1) unidade animal por hectare sendo estas três primeiras áreas localizadas na regiáo de Manicoré, AM, e os solos foram classificados como Argissolo Vermelho Alítico plintico exceto o solo de TPA que foi classificado como Argissolo Vermelho-Amarelo distrófico abrúptico (Embrapa 2013).

A área de agrofloresta (Figura 1) localiza-se nas coordenadas 7o $28^{\prime} 29^{\prime \prime}$ S e 63 $02^{\prime} 07^{\prime \prime}$ W, altitude de 63 m sendo cultivada com café, cacau, palmeiras, andiroba e laranjeiras, com cerca de 20 anos de cultivo e a área de cana-de-açúcar nas coordenadas 7o $54^{\prime} 38^{\prime \prime}$ S e $63^{\circ} 14^{\prime} 27^{\prime \prime} \mathrm{W}$, altitude de $70 \mathrm{~m}$ com cultivo de cana queimada por 10 anos de uso.

A área cultivada com mandioca está situada nas coordenadas geográficas: 70 30' 24" S e 63 04'56” W, altitude de $73 \mathrm{~m}$, com 10 anos de cultivo sucessivo, sendo que a mesma foi corrigida, adubada e gradeada apenas no segundo ano de cultivo, a mesma encontrava-se no quarto mês de plantio, sendo estas três ultimas áreas localizadas na regiáo de Humaitá, AM, e os solos foram classificados como Cambissolo Háplico Alitico plintico (Embrapa 2013).

As áreas localizadas na região de Manicoré, AM apresentam solos desenvolvidos a partir de granitos Rondonianos oriundos do Pré-Cambriano Superior (Brasil 1978). Já as áreas localizadas na região de Humaitá, AM, o material de origem é proveniente dos sedimentos aluviais antigos, que são cronologicamente oriundos do Holoceno (Brasil 1978). O clima da regiâo, segundo a classificação de Köppen, é do tipo tropical chuvoso, com um período seco de pequena duraçáo (Am), e temperaturas variando entre $25^{\circ} \mathrm{C}$ e $27^{\circ} \mathrm{C}$, e com precipitaçóes pluviais entre 2.250 e $2.750 \mathrm{~mm}$ anuais, com chuvas concentradas no período de outubro a junho (Brasil 1978).

Nestas áreas foram estabelecidas malhas de 70 x $70 \mathrm{~m}$ abrangendo 0,49 ha. O solo foi amostrado nos pontos de cruzamento da malha, com espaçamentos regulares de 10 


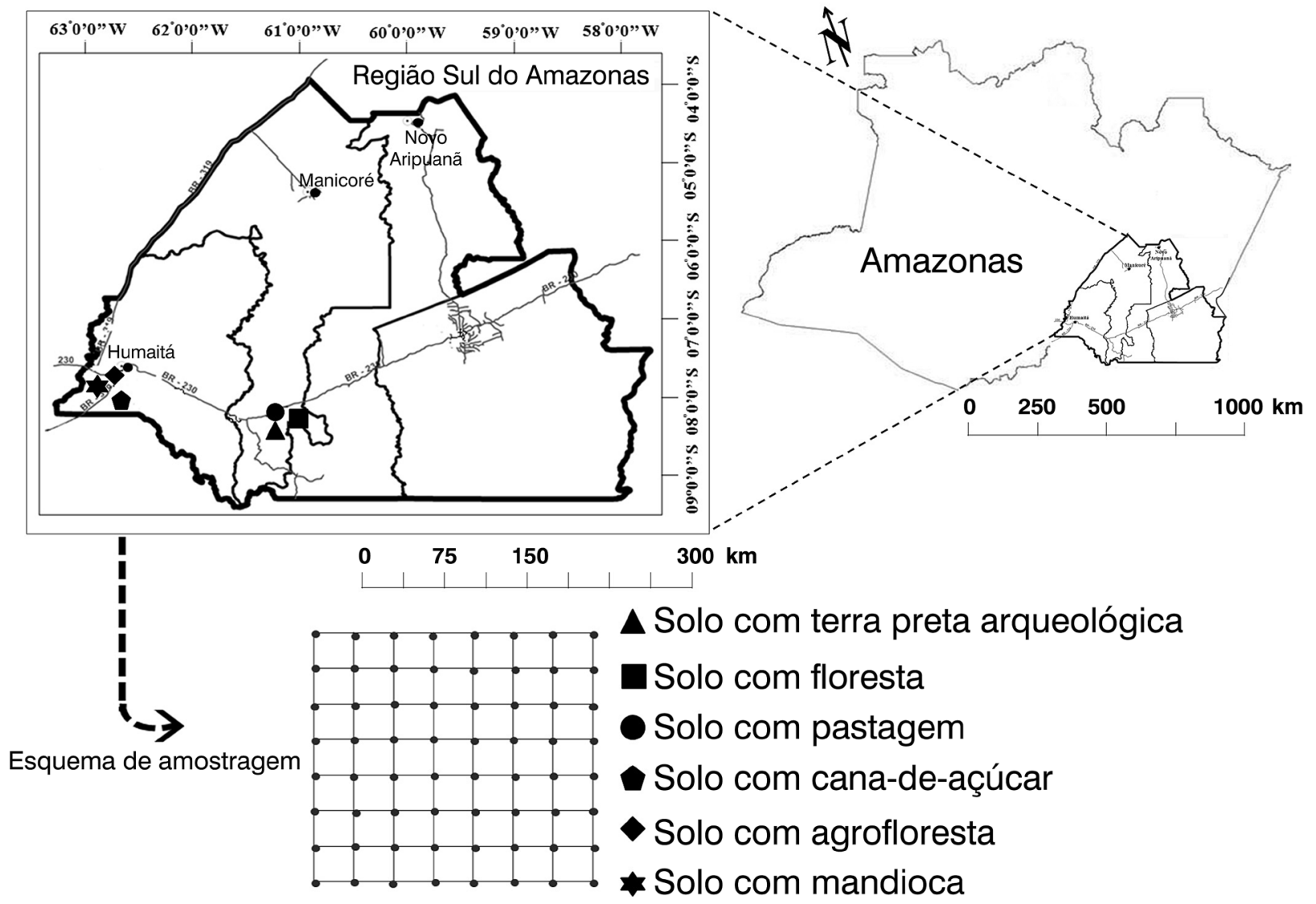

Figura 1. Localização e esquema de amostragem do solo na região Sul do Amazonas.

metros, perfazendo um total de 64 pontos amostrais em cada malha (Figura 1). Em seguida, foi realizada sob cada ponto amostral a coleta de amostras de solos na camada de 0,0-0,10 $\mathrm{m}$ para determinação dos atributos químicos e físicos.

Foram determinados os teores de cálcio $(\mathrm{Ca})$, magnésio $(\mathrm{Mg})$ e potássio $(\mathrm{K})$ trocáveis, fósforo $(\mathrm{P})$ disponível e a acidez potencial $(\mathrm{H}+\mathrm{Al})$ utilizando-se o método da resina trocadora de íons (Raij et al. 2001). O pH foi determinado potenciometricamente com um pHmetro de bancada (W3b, Bel, Araras, SP, Brasil) utilizando-se relação 1:2,5 de solo: em água (Embrapa 2011). O carbono total foi quantificado pelo método de Walkley-Black modificado por Yoemans e Bremner (1988) e a matéria orgânica, por sua vez, foi estimada com base no carbono orgânico. A caracterizaçâo física e química dos solos das áreas estudadas encontra-se na Tabela 1.

Foram coletadas amostras deformadas de solo para análise granulométrica do solo conforme metodologia da Embrapa (2011). Além disso, amostras de solos com estrutura preservada para as análises de macroporosidade (macro), microporosidade (micro), porosidade total $(\mathrm{Pt})$ e densidade do solo (Ds) (Embrapa 2011), por meio de anel volumétrico com $0,045 \mathrm{~m}$ de altura e $0,0405 \mathrm{~m}$ de diâmetro, ao qual, foram coletadas no centro da camada amostrada. Em cada ponto amostral, recolheram-se amostras com estrutura preservada em forma de torráo para determinação da estabilidade dos agregados do solo segundo Kemper e Chepil (1965) e os resultados foram expressos em percentual dos agregados retidos nas peneiras $>2,0 ; 2,0-1,0$; e $<1,0 \mathrm{~mm}$, diâmetro médio geométrico (DMG) e diâmetro médio ponderado (DMP).

A umidade gravimétrica no solo foi determinada com amostras deformadas, conforme o proposto em Embrapa (2011). Na determinação da resistência do solo à penetração, amostras indeformadas de solo em anéis foram coletadas e as medidas tomadas por meio de um penetrômetro eletrônico estático de laboratório (MA933, Marconi, Piracicaba, SP, Brasil) com velocidade constante de penetração de $0,01 \mathrm{~m} \mathrm{~min}^{-1}$, registro de uma leitura por segundo, cone com semiângulo de $30^{\circ}$ e área de $3,14 \mathrm{~cm}^{2}$, equipado com atuador linear e célula de carga de 20 kgf acoplada a um microcomputador para aquisiçáo dos dados, conforme descrito por Tormena et al. (1998). 
Tabela 1. Caracterização física e química de amostras de solo na profundidade de 0,0-0,10 m das áreas estudadas. TPA= terra preta de arqueológica; RSP= resistência do solo a penetração; $\mathrm{Ds}=$ densidade do solo; $\mathrm{Pt}=$ porosidade total; $\mathrm{DMG}=$ diâmetro médio geométrico; $\mathrm{DMP}=$ diâmetro médio ponderado; $>2,00$ mm $(\%)=$ classe de agregados maior que 2,00 milímetros; classe de agregados entre 2,00 e 1,00 milímetros; <1,00 mm (\%)= classe de agregados menor que 1 milímetros. $\mathrm{H}+\mathrm{Al}=$ acidez potencial; $\mathrm{MO}=$ matéria orgânica; $\mathrm{P}=$ fósforo; $\mathrm{K}=$ potássio; $\mathrm{Ca}=$ cálcio; $\mathrm{Mg}$ = magnésio.

\begin{tabular}{|c|c|c|c|c|c|c|c|}
\hline \multirow{2}{*}{ Variáveis } & \multirow{2}{*}{ Unidades } & \multicolumn{6}{|c|}{ Manejos } \\
\hline & & TPA & Floresta & Pastagem & Cana-de-açúcar & Agrofloresta & Mandioca \\
\hline Areia & $\mathrm{g} \mathrm{kg}^{-1}$ & 359 & 411 & 241 & 221 & 159 & 359 \\
\hline Silte & $\mathrm{g} \mathrm{kg}^{-1}$ & 313 & 228 & 474 & 230 & 559 & 313 \\
\hline Argila & $\mathrm{g} \mathrm{kg}^{-1}$ & 328 & 362 & 285 & 549 & 282 & 328 \\
\hline Umidade & $\mathrm{kg} \mathrm{kg}^{-1}$ & 21 & 24 & 26 & 31 & 14 & 21 \\
\hline RSP & Mpa & 2,55 & 2,96 & 2,17 & 4,48 & 1,74 & 2,55 \\
\hline Ds & $\mathrm{kg} \mathrm{dm}^{-3}$ & 1,30 & 1,31 & 1,27 & 1,31 & 1,16 & 1,30 \\
\hline Macro & $m^{3} m^{3}$ & 21 & 17 & 7 & 3 & 13 & 21 \\
\hline Micro & $m^{3} m^{3}$ & 20 & 21 & 29 & 31 & 27 & 20 \\
\hline Pt & $\%$ & 41 & 38 & 36 & 34 & 40 & 41 \\
\hline DMG & $\mathrm{mm}$ & 2,27 & 2,79 & 2,55 & 2,04 & 2,29 & 2,27 \\
\hline DMP & $\mathrm{mm}$ & 2,83 & 3,13 & 3,08 & 2,76 & 2,97 & 2,83 \\
\hline$>2,00$ & $\mathrm{~mm}(\%)$ & 76,38 & 90,52 & 89,64 & 80,79 & 85,07 & 76,38 \\
\hline $2,00-1,00$ & mm(\%) & 9,44 & 3,24 & 2,12 & 4,04 & 3,59 & 9,44 \\
\hline$<1,00$ & $\mathrm{~mm}(\%)$ & 14,18 & 6,24 & 8,24 & 9,03 & 11,34 & 14,18 \\
\hline $\mathrm{pH}$ & $\mathrm{H}_{2} \mathrm{O}$ & 3,98 & 4,30 & 4,40 & 3,80 & 3,91 & 3,98 \\
\hline $\mathrm{H}+\mathrm{Al}$ & $\mathrm{mmol}_{\mathrm{c}} \mathrm{dm}^{-3}$ & 75 & 61 & 89 & 189 & 158 & 75 \\
\hline MO & $\mathrm{g} \mathrm{dm}^{-3}$ & 18,66 & 27,50 & 30,50 & 20,60 & 27,69 & 18,66 \\
\hline$P$ & $\mathrm{mg} \mathrm{dm}{ }^{-3}$ & 6,09 & 4,60 & 6,40 & 8,20 & 6,03 & 6,09 \\
\hline K & $\mathrm{mmol}_{\mathrm{c}} \mathrm{dm}^{-3}$ & 1,66 & 1,71 & 0,49 & 1,11 & 1,40 & 1,66 \\
\hline $\mathrm{Ca}$ & $\mathrm{mmol}_{\mathrm{c}} \mathrm{dm}^{-3}$ & 4,77 & 10,30 & 14,60 & 2,40 & 2,38 & 4,77 \\
\hline $\mathrm{Mg}$ & $\mathrm{mmol}_{\mathrm{c}} \mathrm{dm}^{-3}$ & 2,52 & 4,60 & 8,10 & 1,40 & 1,39 & 2,52 \\
\hline
\end{tabular}

Considerando-se a estrutura multivariada contida nos dados, foram empregadas técnicas estatísticas para verificar semelhanças entre os manejos na tentativa de agrupá-los usando-se os atributos físicos e químicos. Foi realizada a análise de agrupamento por método hierárquico, usando a distância euclidiana como medida de semelhança entre os registros e o método de Ward, como estratégia de agrupamento. $\mathrm{O}$ resultado da análise foi apresentado em forma gráfica (dendrograma) que auxiliou na identificação dos agrupamentos dos ambientes com as variáveis analisadas.

A análise de agrupamento foi complementada com a de componentes principais, para obter um conjunto menor de combinaçóes lineares das variáveis que preservasse a maior parte da informação fornecida pelas variáveis originais (Silva et al., 2010). Esta análise possibilita avaliar como os atributos interagem qualitativamente ao mesmo tempo, cujos valores originais foram normalizados para média igual 0 e variância igual a 1, a fim de compor as variáveis utilizadas na Análise de Componente Principal (ACP). O critério adotado para a escolha do número de componentes foi selecionar aquelas que apresentaram autovalores acima de 1,00 e conseguiram sintetizar uma variância acumulada acima de 70\% (Hair et al. 2005).
Posteriormente, foi realizada uma análise de fatores que permite relaçóes entre um conjunto de variáveis a serem explicadas em termos de um número limitado de novas variáveis e, optou-se por utilizar a extração por componentes principais (Jeffers 1978) calculado a partir da matriz de correlação entre variáveis. Para facilitar a interpretaçáo, utilizou-se o método de rotaçáo ortogonal denominado Varimax, o qual transforma os coeficientes dos fatores de maneira que os valores das correlaçôes entre os fatores e as $n$ variáveis originais se aproximem de zero ou de 1 (Hoffmann 1992).

A fim de comparar os perfis das diferentes áreas utilizando todas as variáveis conjuntamente, foi realizada uma análise complementar que é a análise discriminante, uma matriz de classificação de dados. A partir desta análise foi possível definir o quão distintas são as áreas estudadas, sendo estas representadas em um gráfico, no qual é possível verificar a separaçáo entre elas. Os eixos deste gráfico representam as variáveis canônicas, que são novas variáveis multivariadas criadas a partir do conjunto original de variáveis ou atributos. Todas as análises estatísticas foram processadas com auxílio do programa estatístico Statistica 7.0 (Statistica 2005). 


\section{RESULTADOS}

Com a análise de agrupamento hierárquico para o conjunto de variáveis estudadas, foi possível a divisão de grupos (Figura 2). A divisão mostrou a ordenação dos acessos segundo as características dos atributos do solo. Nesta análise, as áreas TPA, floresta, pastagem, cana-de-açúcar, mandioca e agrofloresta foram agrupadas com base no seu grau de semelhança, classificando-as em grupos homogêneos.

Os autovalores obtido da matriz de covarâncias com valor superior a um (1) (Manly 2008), vem a confirmar a análise de agrupamento para os ambientes estudados. Neste caso, pode-se utilizar o primeiro, o segundo, terceiro e quarto componente principal foram necessárias para explicar no mínimo $70 \%$ da variância total, devido somente estas apresentarem autovalores acima de um (1) (9,70 na $\mathrm{CP} 1 ; 3,00$ na CP2; 2,72 na CP3 e 1,87 na CP4) e com isso, pode-se obter confiança na tomada de decisão quanto aos números de componentes utilizadas. Quanto ao percentual de variância explicada pelas CPs, verifica-se que, as quatro primeiras componentes são responsáveis por $82,47 \%$ da variância total, sendo 46,23 \% na CP1, 14,32\% na CP2, 12,96 na CP3 e 8,94 na CP4.

A análise de componentes principais dos atributos do solo vem a confirmar a análise de agrupamento para os ambientes estudados (Tabela 2 e Figura 3), com a formação de três grupos, na qual a área de floresta, pastagem, cana-de-açúcar e mandioca se encontram mais próxima e mais afastada da área de TPA e agrofloresta, que correspondem à diferenciação dos três ambientes.

A correlação das variáveis (Tabela 2) permitiu caracterizar as variáveis que mais discriminaram na formação e diferenciação dos ambientes. Já para a apresentação gráfica das componentes principais, utilizou-se as duas primeiras componentes principais, para representar o poder discriminatório dos atributos do solo, em cada ambiente estudado. Isso foi devido somente a estas apresentarem elevados autovalores $(9,70$ e 3,00).

A análise de fatores (Tabela 2) explica 46,23\% no primeiro fator, o segundo e o terceiro explicam $14,32 \%$ e $12,96 \%$ respectivamente. Portanto, $73,51 \%$ da variabilidade dos dados é explicada por três fatores principais. Isso mostra que de 21 variáveis, passam-se a utilizar três fatores, com 64 observaçóes que representam o conjunto original, havendo, redução de dimensionalidade das variáveis originais, com perda de explicação de menos de 30\% (26,49\%).

Com o objetivo de verificar as associaçôes entre os ambientes e seus atributos químicos e físicos foi aplicado à técnica complementar com o uso de análise discriminante (Figura 4 e Tabela 3), o que permitiu classificar com eficiência, os resultados obtidos para todas as áreas. Pela análise discriminante, $63,55 \%$ da variabilidade das relaçóes estudadas foram explicados nas duas primeiras raízes. A primeira raiz canônica (Can 1) apresentou 47,35\%, e a segunda (Can 2) 16,20\%. A área com TPA é totalmente diferente dos demais manejos se analisarmos o eixo da Can 1.

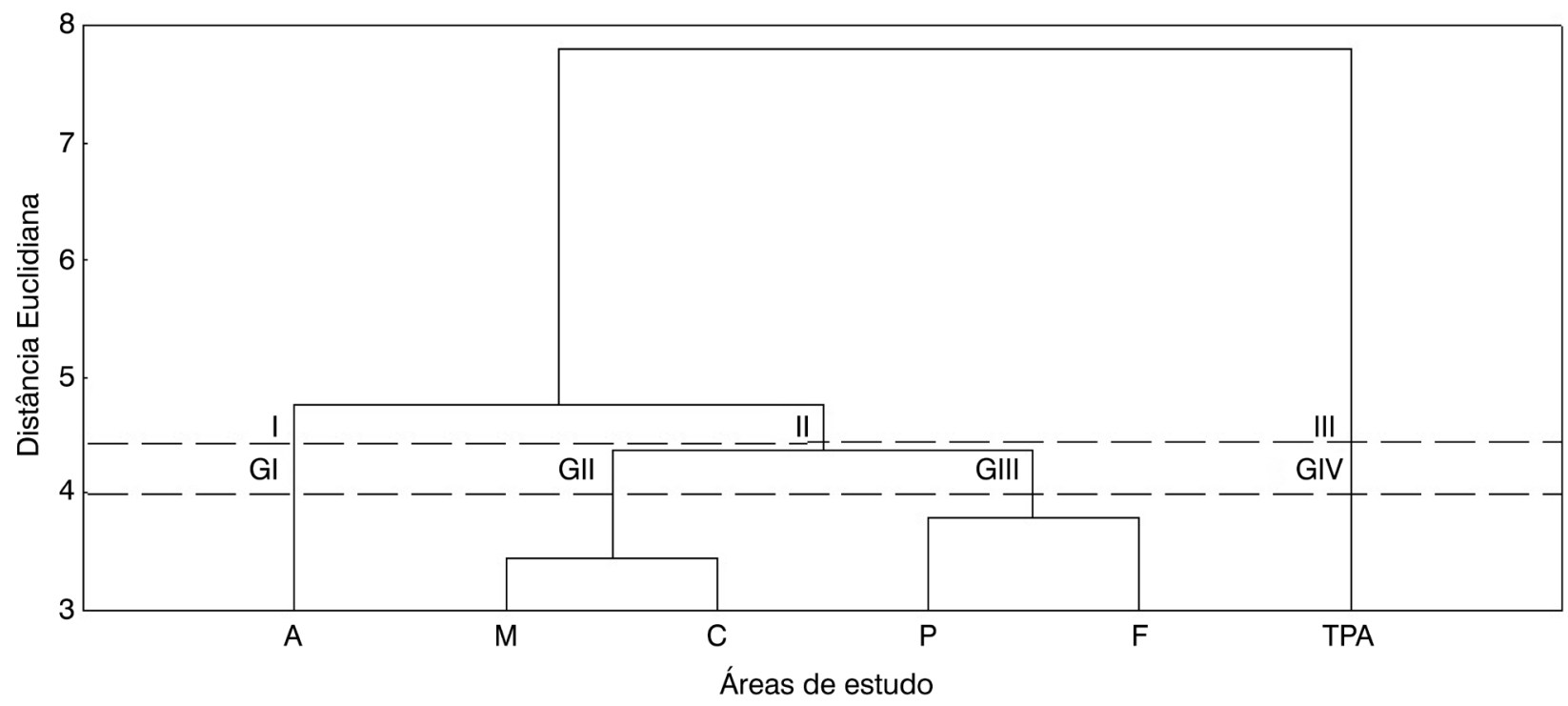

Figura 2. Dendrograma resultante da análise hierárquica de agrupamentos mostrando a formação de grupos segundo as variáveis analisadas dos solos na região Sul do Amazonas. TPA = terra preta arqueológica; $P=$ pastagem; $F=$ floresta; $C=$ cana-de-açúcar; $M=$ Mandioca; $A=$ Agrofloresta. 


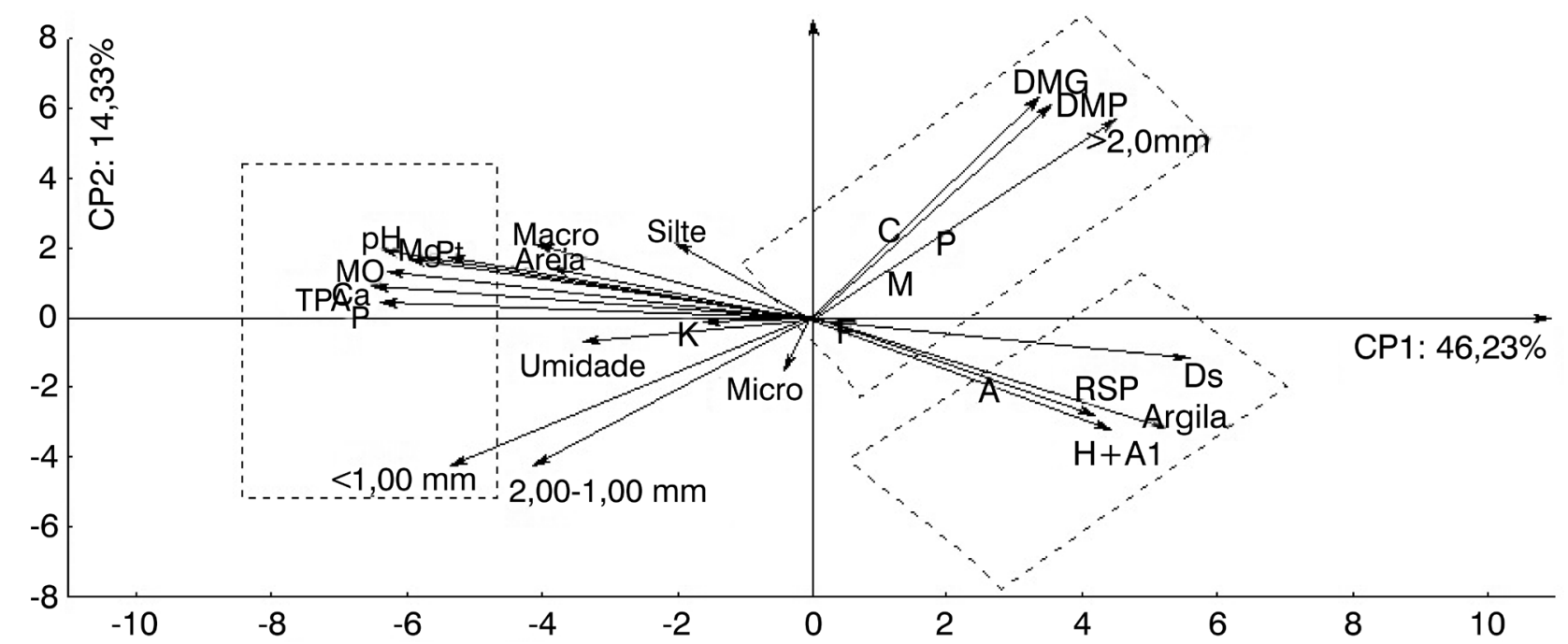

Figura 3. Análise de componentes principais das médias dos atributos dos solos na região Sul do Amazonas. TPA= terra preta arqueológica; $P=$ pastagem; $F=$ floresta; $C=$ cana-de-açúcar; $M=$ Mandioca; $A=$ Agrofloresta. $R S P=$ resistência do solo a penetração; $D s=$ densidade do solo; Macro= macroporosidade; Micro = microporosidade; $\mathrm{Pt}=$ porosidade total; $\mathrm{DMG}=$ diâmetro médio geométrico; $\mathrm{DMP}=$ diâmetro médio ponderado; $>2,00 \mathrm{~mm}(\%)=$ classe de agregados maior que 2,00 milímetros; classe de agregados entre 2,00 e 1,00 milímetros; $<1,00 \mathrm{~mm}(\%)=$ classe de agregados menor que 1 milímetros; $M 0=$ matéria orgânica; $\mathrm{P}=$ fósforo; $\mathrm{K}=$ potássio; $\mathrm{Ca}=$ cálcio; $\mathrm{Mg}=$ magnésio.

Tabela 2. Correlação entre cada componente principal e variáveis analisadas e análise fatorial dos atributos do solo com os fatores (Fator 1, 2, 3 e 4) correspondentes nos ambientes estudados na região Sul do Amazonas. $\mathrm{RSP}=$ resistência do solo a penetração; $\mathrm{DMG}=$ diâmetro médio geométrico; $\mathrm{DMP}=$ diâmetro médio ponderado; $>2,00 \mathrm{~mm}(\%)=$ classe de agregados maior que 2,00 milímetros; classe de agregados entre 2,00 e 1,00 milímetros; $<1,00 \mathrm{~mm}(\%)=$ classe de agregados menor que 1 milímetros; $\mathrm{H}+\mathrm{Al}=$ acidez potencial; $\mathrm{MO}=$ matéria orgânica; $\mathrm{P}=$ fósforo; $\mathrm{K}=$ potássio; $\mathrm{Ca}=$ cálcio; $\mathrm{Mg}=$ magnésio.

\begin{tabular}{|c|c|c|c|c|}
\hline \multirow{2}{*}{ Atributos } & \multicolumn{3}{|c|}{ Fatores } & \multirow[b]{2}{*}{ Fator 4} \\
\hline & Fator 1 & Fator 2 & Fator 3 & \\
\hline Umidade & $-0,481838$ & $-0,087521$ & 0,270496 & $-0,659742$ \\
\hline RSP & 0,622839 & $-0,364545$ & $-0,079526$ & $-0,459556$ \\
\hline Ds & 0,822981 * & $-0,144730$ & $-0,264468$ & $-0,140944$ \\
\hline Macroporosidade & $-0,578994$ & 0,247347 & $-0,630885$ & 0,264027 \\
\hline Microporosidade & $-0,056128$ & $-0,175718$ & $0,908848 *$ & $-0,162358$ \\
\hline Porosidade total & $-0,797008^{*}$ & 0,187469 & $-0,125250$ & 0,219451 \\
\hline Areia & $-0,553339$ & 0,136101 & $-0,620726$ & $-0,406466$ \\
\hline Silte & $-0,288506$ & 0,263311 & 0,555538 & 0,668284 \\
\hline Argila & $0,771825^{\star}$ & $-0,387145$ & $-0,035986$ & $-0,335913$ \\
\hline DMG & 0,502899 & $0,772889 *$ & $-0,111482$ & $-0,139142$ \\
\hline DMP & 0,523622 & $0,755675^{\star}$ & 0,007311 & $-0,080178$ \\
\hline$>2,00 \mathrm{~mm}$ & 0,663161 & 0,696658 & 0,124268 & $-0,110722$ \\
\hline $2,00-1,00 \mathrm{~mm}$ & $-0,586630$ & $-0,521435$ & $-0,390263$ & 0,136642 \\
\hline$<1,00 \mathrm{~mm}$ & $-0,756417^{*}$ & $-0,522880$ & $-0,067543$ & 0,206234 \\
\hline $\mathrm{pH}$ & $-0,912145^{\star}$ & 0,215883 & 0,122544 & $-0,221141$ \\
\hline $\mathrm{H}+\mathrm{Al}$ & 0,654638 & $-0,398125$ & 0,419705 & 0,138848 \\
\hline MO & $-0,859438^{*}$ & 0,200828 & 0,277680 & $-0,171258$ \\
\hline$P$ & $-0,914228$ * & 0,057747 & 0,184600 & $-0,208187$ \\
\hline K & $-0,223613$ & $-0,023332$ & $-0,308466$ & $-0,023403$ \\
\hline $\mathrm{Ca}$ & $-0,936338^{*}$ & 0,100735 & 0,175108 & $-0,223500$ \\
\hline $\mathrm{Mg}$ & $-0,899009^{*}$ & 0,154493 & 0,158816 & $-0,230325$ \\
\hline Variância relativa & $46,23 \%$ & $14,32 \%$ & $12,96 \%$ & $8,95 \%$ \\
\hline
\end{tabular}

* Valores mais discriminatórios.
Tabela 3. Valores resultantes da análise discriminante canônica, evidenciando 0 valor discriminatório de cada atributo dos solos na região Sul do Amazonas. $\mathrm{RSP}=$ resistência do solo a penetração; $\mathrm{DMG}=$ diâmetro médio geométrico; $\mathrm{DMP}=$ diâmetro médio ponderado.

\begin{tabular}{|c|c|c|}
\hline Variáveis & Raiz 1 & Raiz 2 \\
\hline Umidade & $-0,02$ & $-0,04$ \\
\hline RSP & 0,08 & 0,17 \\
\hline Densidade do solo & 0,25 & $-0,22$ \\
\hline Macroporosidade & $-15,23^{*}$ & $73,05^{\star}$ \\
\hline Microporosidade & $-8,44^{*}$ & $40,79^{*}$ \\
\hline Porosidade total & $12,25^{\star}$ & $-58,82^{\star}$ \\
\hline Areia & $-1352,60$ * & $-1637,21^{*}$ \\
\hline Silte & $-1448,27^{*}$ & $-1753,09 *$ \\
\hline Argila & $-1170,14^{*}$ & $-1416,34^{*}$ \\
\hline DMG & $-0,10$ & $-0,06$ \\
\hline DMP & $-0,08$ & $-0,10$ \\
\hline$>2,00 \mathrm{~mm}$ & 0,14 & $-0,43$ \\
\hline $2,00-1,00 \mathrm{~mm}$ & $-0,12$ & $-0,27$ \\
\hline$<1,00 \mathrm{~mm}$ & 0,03 & $-0,21$ \\
\hline $\mathrm{pH}$ & $-0,39$ & 0,49 \\
\hline Acidez potencial & $-0,18$ & 1,21 \\
\hline Matéria Orgânica & 0,44 & $-0,66$ \\
\hline Fósforo & $-0,19$ & $-0,01$ \\
\hline Potássio & 0,18 & 0,01 \\
\hline Cálcio & $-0,75$ & 0,38 \\
\hline Magnésio & $-0,33$ & 0,15 \\
\hline
\end{tabular}

* Caracterização principal das relações canônicas. 


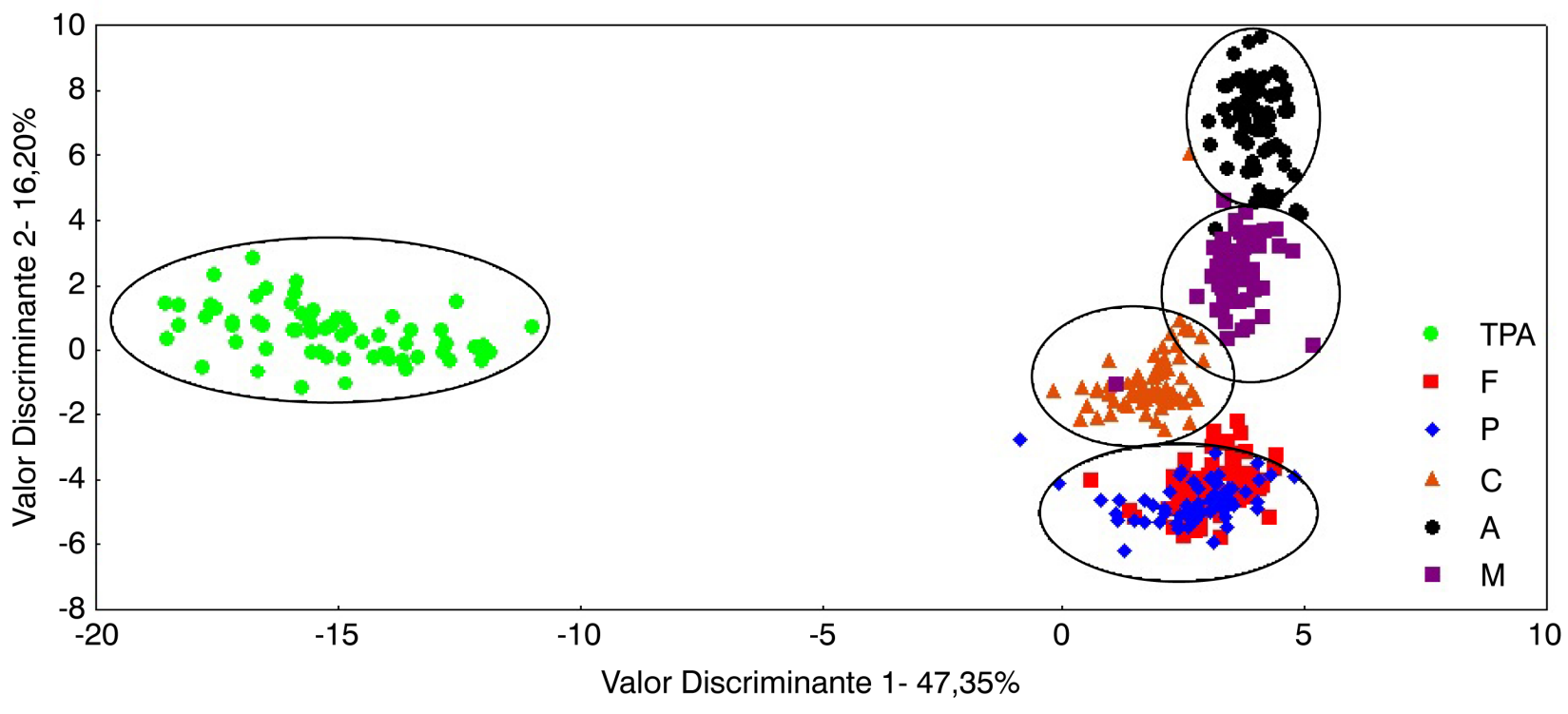

Figura 4. Agrupamento das áreas pela Análise Discriminante Canônica 1 e 2 dos atributos químicos e físicos dos solos na região Sul do Amazonas. TPA= terra preta arqueológica; $\mathrm{P}=$ pastagem; $\mathrm{F}=$ floresta; $\mathrm{C}=$ cana-de-açúcar; $\mathrm{M}=$ Mandioca; $\mathrm{A}=$ Agrofloresta. Esta figura é colorida na versão eletrônica.

\section{DISCUSSÃO}

$\mathrm{Na}$ análise de agrupamento, foi admitido um corte na distância de euclidiana de quatro permitindo uma divisão clara de grupos. Isso indica que usando conjuntamente os atributos químicos e físicos foi possível ordenar os dados em quatro grupos: o GI, englobando os dados que representam a área com agrofloresta, o GII formado pela área mandioca e canade-açúcar, o GIII formado pela área de floresta e pastagem e o GIV formado pela área de TPA, evidenciando, que as áreas possuem diferenças em relaçáo aos atributos analisados.

A diferenciação dos grupos foi marcante, pois as características dos atributos de um mesmo grupo são semelhantes e diferentes do comportamento de outros agrupamentos (Valladares et al. 2008). Esse resultado devese ao fato de que os solos com horizonte antrópico ou terra preta arqueológica (TPA) se diferem química e fisicamente de solos que náo apresentam horizonte antrópico. Campos et al. (2012) afirmam que as TPAs apresentam atributos químicos superiores aos solos não antropogênicos, conferindo-lhes maior fertilidade. Por outro lado, a área de agrofloresta, apresenta-se como ambiente diferenciado, pois os sistemas agroflorestais são reconhecidos por seus efeitos positivos para a conservação e para a biodiversidade do solo, uma vez que a estrutura do sistema agroflorestal aproxima-se de uma vegetação nativa (Brown et al. 2006).

Segundo Young e Hammer (2000), esse comportamento diferenciado dos solos ocorre em funçáo de sua natureza genética, confirmando a premissa da análise de agrupamento, que tem por finalidade reunir, por algum critério de classificação, as unidades amostrais em grupos, de tal forma que exista homogeneidade dentro do grupo e heterogeneidade entre grupos.

No entanto, estas áreas mostram comportamento diferenciado, quando se admite um corte na distância euclidiana 4,5 (Figura 2), formando três grupos, sendo I o grupo formado pela área com agrofloresta, II grupo formado pela área de floresta, pastagem, cana-de-açúcar e mandioca e III o grupo formado pela área de TPA, o que pode ser um indicativo, de que o manejo pode influenciar neste comportamento.

Quanto ao percentual de variância explicado pelas CPs no método dos autovalores, verifica-se que o primeiro, segundo, terceiro e quarta componentes são responsáveis por $82,47 \%$ da variância total, sendo $46,24 \%$ na CP1, $14,33 \%$ na CP2 e 12,96 na CP3 e 8,946\% na CP4, sendo esses valores, atribuídos a variabilidade destes atributos (Loss et al. 2009; Kummer et al. 2010). Esse tipo de análise é interessante para a ciência do solo porque pode eleger as variáveis mais significativas nos processos pedogenéticos (Theocharopoulos et al. 1997). Assim, os atributos do solo analisados com a ACP são agrupados de acordo com suas semelhanças, nas áreas estudadas, que, por sua vez, são separadas por critérios de dissimilaridade, o que levará à formação de grupos.

As variáveis mais fortemente correlacionadas com a área de TPA foram $\mathrm{pH}, \mathrm{MO}, \mathrm{Ca}, \mathrm{Mg}$, P, Pt e classe de agregados $<1,00$ mm (Figura 3), apresentando-se, no terceiro quadrante, com pequeno ângulo em relação ao eixo das abscissas, pois estes 
atributos apresentaram valor absoluto superior a 0,7 . Solos com TPA apresentam maior teor de MO, o que lhe confere maior fertilidade. Altos valores de P é uma característica marcante das TPAs. Em sítios arqueológicos no Amazonas, Cunha et al. (2009) encontraram valor de P de $116 \mathrm{mg} \mathrm{kg}^{-1}$ em áreas de TPA sob floresta, $290,5 \mathrm{mg} \mathrm{kg}^{-1}$ em TPA cultivada e 4,7 $\mathrm{mg} \mathrm{kg}^{-1} \mathrm{em}$ áreas não antropogênicas. A incorporaçáo de resíduos orgânicos, especialmente espinhas de peixe, carapaças de quelônios, ossos de animais ou de seres humanos, ricos em $\mathrm{P}$, contribuiu para o seu elevado teor nas TPAs (Lima et al. 2002).

Os valores de $\mathrm{Ca}$ e $\mathrm{Mg}$ são elevados em TPAs como observados por Barros et al. (2012), justificando sua relaçáo a este ambiente. Isso deve-se à incorporação de ossos ricos em cálcio pelos povos pré-colombianos ou pela maior afinidade do Ca pelas superfícies de troca, resultando em sua menor lixiviaçáo. Campos et al. (2012), encontraram valores de $\mathrm{Ca}$ e $\mathrm{Mg}$ mais elevados nas TPAs, em relaçáo a solos náo antropogênicos.

Quanto a Pt na TPA, pode estar relacionada a presença de fragmentos cerâmicos neste ambientes, que podem contribuir para a presença de partículas de maior tamanho, como o caso da fração areia. Segundo Germam (2003), a presença de pequenos fragmentos líticos e cerâmicos pode contribuir para elevados teores de areia em áreas de TPAs. Os atributos areia e macroporosidade se encontram na terceira componente principal, e de acordo com a direção dos vetores e do ângulo destes em relaçáo ao eixo das abscissas, estes atributos estáo mais relacionados com a TPA, o que é um indicativo de maior Pt nesta área.

As áreas de pastagem, cana-de-açúcar, mandioca e floresta são caracterizadas, por apresentar maior relaçáo com DMG, DMP, porcentagem de agregados, na classe $>2,00 \mathrm{~mm}$ (Tabela 2 e Figura 3). Áreas com floresta apresentam porcentagem de agregados de maior classe, DMG e DMP, pois segundo Salton et al. (2008), a atividade do sistema radicular das plantas, associado à ausência de revolvimento do solo, contribui efetivamente para formação de macroagregados estáveis. As áreas manejadas, como as pastagens, podem apresentar maior agregação, devido à compactação do solo pela maior pressão recebida com o pisoteio animal. Por outro lado, o efeito das pastagens na agregação do solo é atribuído ao crescimento e à atividade do sistema radicular das gramíneas (Silva e Mielniczuk 1997; Liu et al. 2005). Na pastagem a agregação provavelmente está relacionada com a maior ocorrência de ciclos e umedecimento e secagem, devido a pouca cobertura do solo, ocasionando maior exposiçấo aos raios solares (Portugal et al. 2010).

Áreas com cana-de-açúcar em geral, apresentam maior porcentagem de agregados nas classes $>2,00 \mathrm{~mm}$, conforme Souza et al. (2005), que ao avaliarem sistemas de colheita de cana-de-açúcar em um Latossolo Vermelho-Amarelo na região de Ribeirâo Preto, SP, verificaram que a colheita de cana sem queima e com incorporação parcial dos resíduos proporcionou maiores valores de DMP, até $30 \mathrm{~cm}$ de profundidade. Para a área de cana-de-açúcar e de mandioca, esse aumento no tamanho dos agregados pode ter ocorrido também por causa da maior pressão recebida nesse solo em razão da mecanização (Oliveira et al. 2013).

A agrofloresta apresenta forte ligação com a H+Al, RSP, argila e Ds (Tabela 2 e Figura 3). A relação da acidez potencial $(\mathrm{H}+\mathrm{Al})$ em solos mais argilosos é devido a decomposição da matéria orgânica e dos resíduos vegetais, o que leva a liberaçấo de compostos orgânicos na superfície do solo, ocasionando formação de complexos orgânicos hidrossolúveis entre $\mathrm{Ca}$ e Mg com ligantes orgânicos (Miyazawa et al. 1993), facilitando a descida desses cátions no perfil do solo (Franchini et al. 1999), o que ocasiona a acidificação do solo. Em relaçấo à RSP, esta é influenciada pela textura, conteúdo de água, densidade do solo e tipo de mineral de argila no solo (Gomes e Peña 1996). Nesse contexto, o conteúdo de água na capacidade de campo é considerado ideal para a determinação da RSP, condiçáo em que é obtida melhor correlação com a densidade do solo (Henderson 1989). Beutler et al. (2007), encontraram correlação positiva entre a RSP e Ds na comparação de penetrômetros usados para a avaliação da compactação de Latossolos.

$\mathrm{Na}$ análise de fatores, as cargas fatoriais que definem quais variáveis são mais importantes, indicam que as variáveis que formam o fator $1, \mathrm{Pt}$, argila, agregados na classe $<1,00 \mathrm{~mm}, \mathrm{pH}, \mathrm{Ca}, \mathrm{Mg}, \mathrm{MO}, \mathrm{P}$ e Ds; no fator 2 DMG e DMP; e no fator 3 a Pt que possuem cargas fatoriais elevadas (Tabela 2). Estas variáveis explicam a maior porcentagem de variação e são as que mais contribuem para alterar as características do solo em funçấo do manejo. As variáveis que apresentaram cargas fatoriais abaixo desses valores são aquelas que retêm pequena parte da variaçáo total, o que demonstra que as demais variáveis não citadas contribuem em menor proporção para discriminar o uso do solo (Pragana et al. 2012).

Os resultados da análise discriminante demonstram que a Can 1 é responsável pela separação da TPA das outras áreas, sendo este solo, com melhor qualidade física e química, apresentando características mais distante dos demais ambientes. Esta separação de ambientes está de acordo com a análise de componentes principais (Figura 3), e com a análise de agrupamento. Oliveira (2013), avaliando conjuntamente os atributos físicos, químicos e a suscetibilidade magnética do solo na caracterização de três ambientes na região sul do Amazonas (floresta, pastagem e TPA) apresentou resultado semelhante, onde a área com TPA é totalmente diferente dos demais ambientes, com uso de técnicas estatísticas multivariadas.

De acordo com a análise discriminante, os atributos que mais contribuíram para a separação dos ambientes são 
areia, silte, argila, macroporosidade, microporosidade e porosidade total (Tabela 4), enfatizando que os atributos granulométricos possuem maior poder discriminatório, visto que estes solos são de diferentes classes.

A análise de componente principal e a análise discriminante mostram de forma clara a ligação ou as alteraçôes nos solos, quando se aplica um manejo específico. Quando se compara a floresta como um ambiente em equilíbrio com as demais áreas, nota-se que o ambiente de pastagem se encontra mais próximo ou com características mais próximas da área de floresta. Este comportamento pode indicar que o uso da pastagem não causa grandes alteraçôes no solo. Por outro lado, as área de cana-de-açúcar e mandioca estáo mais próximas da floresta, do que a área de TPA e agrofloresta.

A área de agrofloresta, por ser um ambiente em recuperaçáo, suas características se distanciam da área de floresta, pois o tempo de recuperação não foi suficiente para estabelecer um novo equilíbrio e assim, o solo apresentar características próximas a floresta. Por outro lado, a TPA com sua alta fertilidade, é um ambiente diferenciado, mostrando-se maior distância das demais áreas (Figuras 3 e 4).

Neste sentido, avaliando as Figuras 3 e 4, observa-se que pela componente principal, a área de TPA apresentase no terceiro e quarto quadrante, com maior distância da área da floresta. Já a área de floresta, encontra-se nos mesmos quadrantes da área de pastagem, cana-de-açúcar e mandioca, demonstrando que estes ambientes apresentam características mais semelhantes. Dentro deste contexto, avaliando estas áreas pela análise discriminante, observase comportamento semelhante, no entanto, demonstra quais as ligaçóes entre as áreas. A área de pastagem e floresta estão sobrepostas uma a outra, mostrando maior homogeneidade entre os ambientes. Já ás áreas de canade-açúcar e mandioca, apesar de estarem mais afastadas, apresentam características comuns entre elas. A área de agrofloresta, encontra-se mais afastada da área de floresta e das demais áreas, sendo um indicativo de heterogeneidade entre estes ambientes.

Sendo assim, os atributos que podem ser possíveis indicadores da separação dos ambientes são o cálcio, magnésio, fósforo, matéria orgânica, $\mathrm{pH}$ e porosidade total para a área com terra preta arqueológica, DMG, DMP e agregados $>2,00$ $\mathrm{mm}$ para a floresta, mandioca, pastagem e cana-de-açúcar e acidez potencial, argila, resistência a penetração e densidade do solo para a agrofloresta. Estes atributos podem sofrer maiores alteraçôes se submetidos a açôes antrópicas, sendo estes possíveis indicadores da caracterização destes ambientes. $\mathrm{O}$ uso de atributos do solo identificadores de diferentes ambientes é uma ferramenta fundamental para direcionar práticas que reduzam o depauperamento.

\section{CONCLUSÕES}

A área com terra preta arqueológica apresenta maior relação aos atributos químicos, o que confere maior fertilidade. Por serem ambientes manejados, as áreas de mandioca, cana-de-açúcar e pastagem apresentam características físicas e químicas homogêneas. Os atributos químicos foram responsáveis pela separação da terra preta arqueológica e agrofloresta das demais áreas.

\section{AGRADECIMENTOS}

À Fundação de Amparo à Pesquisa do Estado do Amazonas - FAPEAM, Fundaçáo de Amparo à Pesquisa do Estado de São - FAPESP e Secretaria de Estado da Ciência, Tecnologia e Inovação - SECT/AM, pelo financiamento e apoio da pesquisa.

\section{BIBLIOGRAFIA CITADA}

Barros, K. R. M.; Lima, H. V.; Canellas, L. P.; Kern, D.C. 2012. Fracionamento químico da matéria orgânica e caracterização física de Terra Preta de Índio. Revista Ciências Agrária, 55:44-51.

Beutler, A. N.; Centurion, José F.; Silva, A. P. 2007. Comparação de penetrômetros na avaliação da compactação de Latossolos. Engenharia Agricola, 27:146-151.

BRASIL. 1978. Ministério das Minas e Energia. Projeto Radambrasil, folha SB. 20, Purus. Rio de Janeiro, 1978. 561 p.

Brown, G. G.; Römbke, J.; Höfer, H.; Verhaagh, M.; Sautter, K. D.; Santana, D. L. Q. 2006. Biodiversity and function of soil animals in Brazilian agroforestry systems. In: Gama-Rodrigues, A. C.; Barros, N. F.; Gama-Rodrigues, E. F.; Freitas, M. S. M.; Viana, A. P.; Jasmin, J. M.; Marciano, C. R.; Carneiro, J. G. A. (eds.) Sistemas Agroflorestais: Bases Cientificas para o desenvolvimento sustentável. UENF, Campos dos Goytacazes, p.217-242.

Campos, M. C. C. 2009. Pedogeomorfologia aplicada a ambientes Amazônicos do Médio Rio Madeira. Tese de Doutorado, Universidade Federal Rural de Pernambuco, Recife, 260p.

Campos, M. C. C.; Santos, L. A. C.; Silva, D. M. P.; Mantovanelli, B. C.; Soares, M. D. R. 2012. Caracterizaçáo física e química de terras pretas arqueológicas e de solos não antropogênicos na região de Manicoré, Amazonas. Revista Agro@mbiente, 6:102-109.

Cunha, T. J. F.; Madari, B. E.; Canellas, L. P.; Ribeiro, L. P.; Benites, V. M.; Santos, G. A. 2009. Soil organic matter and fertility of anthropogenic dark earths (terra preta de índio) in the brazilian amazon basin. Revista Brasileira de Ciência do Solo, 33:85-93.

Cruz, C. D.; Regazzi, A. J. 2001. Modelos biométricos aplicados ao melhoramento genético. 2.ed. rev. Viçosa: UFV, 2001,390p.

Embrapa. 2011. Centro Nacional de Pesquisa de Solos. Manual de métodos de análise de solo. Rio de Janeiro, 2011.230p.

Embrapa. 2013. Sistema Brasileiro de Classificação de Solos. Centro Nacional de Pesquisa de Solos. 3.ed. revisada e ampliada. Brasília. 2013. 353p.

Franchini, J. C.; Miyasawa, M.; Pavan, M. A.;Malavolta, E. 1999. Dinâmica de íons em solo ácido lixiviado com extratos de 
resíduos de adubos verdes e soluçôes puras de ácidos orgânicos. Pesquisa Agropecuária Brasileira, 34:2267-2276.

German, L. A. 2003. Historical contingencies in the coevolution of environment and livelihood: contributions to the debate on Amazonian Black Earth. Geoderma, 111:307-331.

Gomes, A. Da S.; Peña, Y.A. 1996. Caracterização da compactação através do uso do penetrômetro. Lavoura Arrozeira, 49:18-20.

Hair J. R.; Anderson, R. E.; Tatham, R. L.; Black, W. C. 2005. Análise multivariada de dados. Porto Alegre. 2005. 211p.

Henderson, C.W.L. 1989. Using a penetrometer to predict the effects of soil compaction on the growth an yield of wheat on uniform, sandy soils. Australian Journal of Agricultural Research, 40:497-508.

Hoffmann, R. 1992. Componentes principais e análise fatorial. Piracicaba: Departamento de Economia e Sociologia Rural. Escola Superior de Agricultura "Luiz de Queiroz", Universidade de São Paulo, 1992. 25p. (Série Didática n. 76).

Jeffers J.N.R. 1978. An Introduction to System Analysis: with Ecological Applications. E. Arnold Publ., London, 1978.198p

Kämpf, N.; Kern, D.C. 2005. O solo como registro da ocupação humana pré-histórica na Amazônia. In: Vidal-Torrado, P.; Alleoni, L.R.F.; Cooper, M.; Silva, A.P. \& Cardoso, E.J., eds. Tópicos em ciência do solo. Sociedade Brasileira de Ciência do Solo, Viçosa, MG, p. 277-320.

Kemper, W. D.; Chepil, W. S. 1965. Aggregate stability and size distribution. In: BLACK, C. A. (Ed.) Methods of soil analysis. Madison: ASA, 39, 499-510.

Kummer, L.; Melo, V. F.; Barros, Y. J.; Azevedo, J. C. R. 2010. Uso da análise de componentes principais para agrupamento de amostras de solos com base na granulometria e em características químicas e mineralógicas. Scientia Agraria, 11:469-480.

Lima, H. N.; Schaefer, C. E. R.; Mello, J. W. V.; Gilkes, R. J.; Ker, J. C. 2002. Pedogenesis and pre-Columbian land use of "Terra PretaAnthrosols" (Indian black earth") of Western Amazonia. Geoderma, 110:1-17.

Liu, A.; Ma, B. L.; Bomke, A. A. 2005. Effects of cover crops on soil aggregate stability, total organic carbon, and polysaccharides. Soil Science Society of America Journal, 69:2041-2048.

Loss, A.; Pereira, M. G.; Schultz, N.; Anjos, L. H. C.; Silva, E. M. R. 2009. Atributos químicos e físicos de um Argissolo VermelhoAmarelo em sistema integrado de produção agroecológica. Pesquisa Agropecuária Brasileira, 44:68-75.

Manly, B. J. F. 2008. Métodos estatísticos multivariados: uma introdução. 3.ed. Porto Alegre: Bookman, 2008. 229p.

Marçal, C. T.; 2009. Efeitos da cultura da cana-de-açúcar e seu manejo (uso de vinhaça e método de colheita) sobre a mesofauna do solo. Dissertação de Mestrado, Universidade Federal do Paraná, Curitiba, Santa Catarina. 124p.

Macdicken, K,G.;Vergara, N.T. 1990. Agroforestry: classificationand management. New York: John Wiley, 1990. 382p.

Matos,P. L. P.; Bezerra, V. S. 2003. Cultivo da Mandioca para o Estado do Amapá. Embrapa Mandioca e Fruticultura. Sistemas de Produção, 2. (http://sistemasdeproducao.cnptia.embrapa.br/
FontesHTML/Mandioca/mandioca_amapa/index.htm). Acesso em: 19/10/2011.

Miyazawa, M.; Pavan, M. A.; Calegari, A. 1993. Efeito de material vegetal na acidez do solo. Revista Brasileira de Ciência do Solo, 17:411-416.

Oliveira, I. A.; Campos, M. C.C.; Soares, M. D. R.; Aquino, R. E.; Marques Júnior, J.; Nascimento, E. P. 2013. Variabilidade espacial de atributos físicos em um CambissoloHáplico, sob diferentes usos na região sul do Amazonas. Revista Brasileira de Ciência do Solo, 37:1103-1112.

Oliveira, I. A. 2013. Caracterizaçâo de solos sob diferentes ambientes na região Sul do Amazonas. Dissertação de Mestrado, Universidade Estadual Paulista Júlio de Mesquita Filho, Jaboticabal, São Paulo. 105p.

Portugal, A. F.; Costa, O. D. V.; Costa, L. M. 2010. Propriedades físicas e químicas do solo em áreas com sistemas produtivos e mata na região da Zona da Mata Mineira. Revista Brasileira de Ciência do Solo, 34:575-585.

Pragana, R. B; Ribeiro, M.R; Nóbrega, J. C. A; Ribeiro Filho, M.R; Costa, J. A. 2012.Qualidade física de latossolos amarelos sob Plantio direto na região do cerrado piauiense. Revista Brasileira de Ciência do Solo, 36:1591-1600.

Raij, B. Van.; Andrade, J. C.; Cantarella, H.; Quaggio, J. A. 200. Análise química para avaliação da fertilidade do solo. Campinas: Instituto Agronômico. 2001. 285 p.

Salton, J. C.; Mielniczuk, J.; Bayer, C.; Boeni, M.; Conceição, P.C.; Fabrício, C. A.; Macedo, M. C. M.; Broch, D. L. 2008. Agregação e estabilidade de agregados do solo em sistemas agropecuários em Mato Grosso do Sul. Revista Brasileira de Ciência do Solo, 32:11-21.

Silva, I. F.; Mielniczuk, J. 1998. Sistemas de cultivo e características do solo afetando a estabilidade de agregados. Revista Brasileira de Ciência do Solo, 22:311-317.

Silva, M. O.; Freire, M. B. G. S.; Mendes, A. M. S.; Freire, F. J.; Campos, M. C. C.; Amorim, L. B. 2010. Discriminação de diferentes classes de solos irrigados com águas salinas, na região de Mossoró, RN, com o uso de análise multivariada. Ambiência, 2:261-270.

Silva, R. R.; Silva, M. L. N.; Ferreira, M. M. 2005. Atributos físicos indicadores da qualidade do solo sob sistemas de manejo na bacia do Alto do Rio Grande MG. Ciência e agrotecnologia, 29:719-730.

Souza, Z. M.; Prado, R. M.; Paixão, A. C. S.; Cesarin, L. G. 2005. Sistemas de colheita e manejo da palhada de cana-de-açúcar. Pesquisa Agropecuária Brasileira, 40:271-278.

Statistica. 2005. Statistica 7.0, EUA Software. Tucksa: StatSof.

Theocharopoulos, S. P.; Petrakis, P. V.; Trikatsoula, A. 1997. Multivariate analysis of soil grid data as a soil classification and mapping tool: the case study of a homogeneous plain in Vagia, Viotia, Greece. Geoderma, Amsterdam, 77:63-79.

Tormena, C. A.; Silva, A.P.; Libardi, P. L. 1998. Caracterização do intervalo hídrico ótimo de um Latossolo Roxo sob plantio direto. Revista Brasileira de Ciência do Solo, 22:573-581. 
Valladares, G. S.; Gomes, E. G.; Mello, J. C. C. B. S.; Pereira, M. G.; Anjos, L. H. C.; Ebeling, A. G.; Benites, V. M. 2008. Análise dos componentes principais e métodos multicritério ordinais no estudo de Organossolos e solos afins. Revista Brasileira de Ciência do Solo, 32:285-296.

Yoemans, J. C.; Bremner, J. M. 1988. A rapid and precise method for routine determination of organic carbon in soil. Communications in Soil Science and Plant Analysis. 19:1467-1476.
Young, F. J.; Hammer, R. D. 2000. Defining geographic soil bodies by landscape position, soil taxonomy, and cluster analysis. Soil Science Society of America Journal, 64:989-998.

Recebido em 05/02/2014

Aceito em 10/07/2014 
